\title{
Constraints facing surveillance in the Eastern Mediterranean Region
}

2. Hallaj'

The important role of surveillance in reporting, monitoring and responding to infectious diseases has been repeatedly stressed by the World Health Organization. In 1995 the Forty-eighth World Health Assembly in its resolution on the control of emerging infectious diseases re-emphasized these facts and urged Member States to strengthen national programmes of surveillance for infectious diseases and their causative agents. The same resolution requested WHO to draw up plans for improving national and international surveillance of infectious diseases. In order to achieve this objective the national surveillance systems in the WHO Eastern Mediterranean Region were reviewed and analysed in terms of their structure, process and output to identify the main constraints.

Surveillance sys ems in the Region vary widely. In some countries they are good and functional, while in others they are rudimentary and almost nonexistent. However, it is prudent to say that most surveillance systems in the Region face one or more of the following constraints.

\section{Inadequate appreciation of the value of surveillance for decision- making}

The objective of a surveillance system is to support the planning, implementation and evaluation of public health interventions and programmes. Although the final output of the surveillance system may well be a communication or report to the decisionmakers, it is how that information is used that is the ultimate objective of the surveillance system. This is a health policy point of prime importance that does not receive the attention it deserves. In many instances authorities pay lip service without solid commitment. This is reflected in the weakness of the resources allocated to and the organizational set-up of the system, as well as in the use of the information generated by the system for appropriate action.

In general, there is a need for a change of attitude in all of those responsible for decision-making at the national level so that the application of epidemiological principles and approaches, including disease surveillance, will have a significant yield for national health programmes. It is unfortunate that many of those supposed to participate in this process of reorientation of the decisionmakers sometimes play the opposite role in their endeavour to appease the selfsame decision-makers.

\section{Lack of surveillance guidelines as well as lack of clear assignment of responsibilities for all participants in survolllance at all levels}

Few surveillance guidelines are available in the countries of the Eastern Mediterranean Region. In most countries, the scheme for

${ }^{1}$ Regional Adviser, Control of Communicable Diseases, World Health Organization, Regional Office for the Eastern Mediterranean. Alexandria, Egypt.

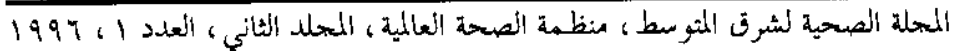


the now of information from its sources to the decision-making level and the feedback mechanism are not usually clear. At the same time responsibilities for surveillance activities at each level of the health system are not well delineated. This leads sometimes to constrictions in the flow of information and in many instances to a "keep a low profile" or "do not do others' jobs" attitude in many health workers, who teel that they are already overburdened with work. Even when guidelines have been developed and produced, many peripheral health centres have not been advised of them or have "locked them in" so that many newcomers to the system have no idea about them.

\section{Lack of standard case definitions for diseases under surveillance}

It is obvious that using the same definition and criteria to diagnose a reportable disease in a country and between countries is a prerequisite for diagnostic accuracy and consistency of data reported by different health facilities. Standard case definitions and criteria for diagnosis are absent in many countries of the Eastem Mediterranean Region, except for those presented by WHO for diseases under specific programmes. It may not be easy to reach a consensus about a standard case definition for several infectious diseases that will satisfy clinicians and be at the same time simple enough to be applied by the majority of lower level health workers. We have noticed several instances of resentment of proposed standard case definitions by top-level clinicians who consider such standardization as a derogation of their clinical skills and refuse to use it or introduce it in the training of their students, even if it is mainly for reporting purposes.
Inabllity to achleve the needed concordance between efficiency, simplicity and sufficiency

The quantity of information collected and reported must balance the need for simplicity, to increase the efficiency of the system, and the need for sufficient data, to increase the usefulness of the system. Certain data are needed in order to describe the occurrence of disease over time, place and person. This balance is not achieved in many systems in the Eastern Mediterranean Region. Data collected are usually of very little real value, and sometimes the information compiled is unnecessary and redundant, hence complicating every step in the surveillance process. This is mostly due to lack of previous determination of the expected indicators to be drawn from the collected data, which in itself reflects a conceptual misunderstanding of the whole surveillance process. This lack of balance results in an incomplete picture of disease patterns in some communities and a waste of time and effort in collecting unneeded data in others.

\section{Lack of community participation}

The community is the centre of interest of all measures for disease prevention and control, including surveillance. No approach can be successful without the full cooperation of the community. Its participation in early disease reporting and in reviewing the situation and its underlying factors, as well as in the implementation of the needed measures for control, are indispensable to ensure the highest quality of surveillance. Community involvement is also a strong force in attracting the needed attention and interest of decision-makers. It has been noticed that in several countries of the Region, community participation in the surveillance system is lacking, although disease reporting in certain areas depends on securing the coopera- 
tion of the community; and failure to achieve full reporting will be reflected in the output of the surveillance process. Lack of community participation stems trom a prevailing attitude in most countries-to look at disease control as a service provided by workers in the health system to a passive receiver, which is the community. The concept and practice of partnership in achieving a common objective seems to be lacking in many people in both the community and the health system.

\section{Weak participation from the private health sector and governmental institutions other than the ministry of health}

One weak spot in the surveillance systems in several countries in the Eastern Mediterranean Region is the limited participation of the private health sector. This is particularly so where the private sector provides a substantial share of the health services. In many countries private physicians are the forefront of the battle against disease, and their input is vital for strengthening surveillance. They could be looked upon as one of the sensitive antennae of the whole system of surveillance. Several attempts have been made to achieve private practice participation, ranging from legislative measures to providing incentives, but none has been truly successful so far. We believe that the appropriate protocol for cooperation between the governmental health system and the private sector is still lacking, and further efforts are needed to develop it. As well, government health institutions other than those following the ministry of health, such as university health institutions or military health services, do not usually collaborate with the national surveillance system, although in many instances they cover a large portion of the population.
Weakness in the capacity to monitor completeness and timeliness of reporting and response

The capacity to monitor the process and the output of the surveillance system, as well as the response triggered by this information, is an important indicator of the strength of the system and its proper functioning. The concept of monitoring health activities is still underrated in so many health services in the Eastern Mediterranean Region. This is very much one of the surveillance systems in particular, whose proper functioning depends to a great extent on supervision and monitoring.

\section{Strong centralization of analysis and Interpretation of data and initiation of action with weak involvement of the periphery}

A strong central unit is needed for effective surveillance. However, this should never hinder the process of analysis and interpretation of data at different levels in the health system, and especially at the peripheral level. In many highly centralized surveillance systems in the Eastern Mediterranean Region, by the time data reach the central level, to be analysed and information for action generated, the time for the action to be effective has already passed. This is a grave weakness, which is more noticeable in countries where proper communication is lacking and peripheral health workers are not trained to analyse and interpret data. This weakness clearly affects the role of surveillance as an early warning system, which is so important in early detection control of emerging infections.

\section{Weakness of the feedback mechanism}

Feedback mechanisms are one of the weakest points in surveillance systems in the

المجهلة الصحية لشرق المتوسط ؛ منظمة الصجهة العالية، المجلد الثاني، العلدد 1، 1999 
Eastern Mediterranean Region. There is usually no way of showing peripheral staff the value and utility of their vital contribution to the system, and hence they loose interest in surveillance activities. Lately, a few countries in the Region have taken relatively successful steps to overcome this constraint by issuing surveillance newsletters or epidemiological bulletins. We hope that this activity will be promoted, maintained and followed up by other countries as a method of feedback.

\section{Weak support from the laboratory}

Weakness in laboratory diagnostic capabilities with respect to infectious diseases is a prominent feature in many national public health laboratories in the Eastern Mediterranean Region. This is mostly due to lack of trained human resources and necessary equipment and supplies. As well, in most instances there are no guidelines or quality control. Poor managerial processes and abilities may lead to inadequate collaboration between laboratory services and disease control departments that is reflected in inefficiency of surveillance support.

\section{Weak envlronmental survelllance activities}

Environmental surveillance activities are another important weakness in the support system. For example, very few data are available on animal reservoirs and types of vectors, their geographic distribution, their habits and level of resistance to insecticides.

\section{Shortage of trained personnel}

One of the most important constraints facing surveillance in many countries of the Eastern Mediterranean Region is the shortage in trained health personnel at all levels in the health system. This is usually due to:

- deficient epidemiological training in the basic education of health workers, whether they be graduates of medicine schools, nursing schools or paramedical institutes;

- lack of a system of continuous in-service training of health workers; and

- scarcity of training modules on disease surveillance at different levels. Several modules have been developed by WHO on surveillance of specific diseases, but these should be further modified to come up with a module on disease surveillance in general. 